\title{
Evaluation of Academic Level of Sci-tech Journals Based on Rough Set and TOPSIS
}

\author{
He Nie ${ }^{1}$, Yuan-Biao Zhang ${ }^{2,3}$, Zhen Zhang ${ }^{4}$, Xin-Guang $\mathrm{Lv}^{2,3}$ \\ ${ }^{1}$ Electrical and Information School, Jinan University, Zhuhai 519070, China \\ ${ }^{2}$ Packaging Engineering Institute, Jinan University, Zhuhai 519070, China \\ ${ }^{3}$ Key Laboratory of Product Packaging and Logistics of Guangdong Higher Education Institutes, Jinan \\ University, Zhuhai 519070, China \\ ${ }^{4}$ International Business School, Jinan University, Zhuhai 519070, China \\ Correspondence: Yuan-Biao Zhang, Packaging Engineering Institute, Jinan University, Zhuhai 519070, China. \\ E-mail: zybt@jnu.edu.cn
}

Received: September 28, 2014 Accepted: October 28, 2014 Online Published: December 19, 2014

doi:10.5539/ijsp.v4n1p12

URL: http://dx.doi.org/10.5539/ijsp.v4n1p12

\begin{abstract}
This paper aims to objectively evaluate the academic level of sci-tech journals, reducing mistakes and random errors caused by human factors in traditional academic evaluation. Evaluation indicators of sci-tech journals are reduced based on equivalence relation thought in Rough set theory, removing the miscellaneous indicators, and form the core evaluation indicator system. By studying the degree of importance of the core evaluation indicators' attributes to determine the appropriate weight, to avoid interference of human factors in the weight determination, so that the evaluation results of sci-tech journals can be more objective. Combine obtained weight of core evaluation indicators with related data, and using TOPSIS method to make comprehensive evaluation rankings for journals. Finally, using the model to validate data of sci-tech journals, and achieved good results, proved the feasibility and effectiveness of the model.
\end{abstract}

Keywords: Academic evaluation, Rough set, TOPSIS

\section{Introduction}

Currently, the academic evaluation for the assessment of academic quality, academic value, identification of academic achievement, and guidance of academic orientation is important, but there are some problems of academic evaluation, Such as the lack of a reasonable academic evaluation, using the academic literature search tool as evaluation criteria, affected by administrative intervention, the disorder of academic evaluation have made the evaluated have a variety of game behavior. To solve these problems, researchers have made many useful exploration and research, proposed various methods. For example, Wang Ling-zhi, et al(2012) use key factors to ensure key indicators in evaluation of academic journals and use TOPSIS to conduct comprehensive rankings of journals. Cao Zhi-gang, et al (2007) apply multilevel comprehensive evaluation method to assessment of scientific research subjects. Chen Han-zhong et al (2003) apply principal component analysis to the evaluation of academic journals. Lin Chun-yan et al (2004) came up with a tribute mathematical model for comprehensive index system' quality evaluation for natural science academic journals

Rough set theory is proposed by professor Pawlak in 1982, which is a mathematical tool can quantitative analyze imprecise, inconsistent, and incomplete information and knowledge (Wang Guo-ying et al., 2009).Li Yuan-yuan (2009) make a research on the construction of indicator system and comprehensive evaluation method based on Rough set. Huang Jian et al (2009) study the degree of importance of the core evaluation indicators' attributes to determine the appropriate weight. Xiao Hua et al (2001) make an evaluation method for science research project based on Rough set. TOPSIS is an excellent evaluation method. Based on the original data sample itself, using Euclidean distance to calculate the distance to the ideal solution, using the comparison fully of the original data, less information loss (WANG Yan-yu et al., 2011). Tao Shu-jin(2013) use the least squares method to determine the indicators of subjective weights and entropy method to determine the objective weight, combining the two weights to determine the final weight matrix, that is, Combination Empowerment-TOPSIS Method.

This paper presents an evaluation model based on Rough sets and TOPSIS method to evaluate the academic 
level of sci-tech journals. Comparing with traditional method about academic evaluation, the method used in this paper can effectively analyze imprecise, inconsistent and incomplete data, fully min the intrinsic link of data obtained and without human experience, making the scientific results of the evaluation more objective and scientific. By using the model to validate data in reference (He De-fang et al., 2013), it shows that the model has good accuracy and applicability.

\section{The Academic Level of Sci-tech Journals Evaluation Model Based on Rough Sets and TOPSIS}

In allusion to academic level of sci-tech journals evaluation, Firstly, the use of relevant academic level of sci-tech journals evaluation indicators data, combined with equivalence relation thought in Rough set theory to establish reduction algorithm for evaluation indicators, which can remove miscellaneous indicators, making the evaluation process more efficient. Then according to the degree of importance of the evaluation indicators' attributes, to obtain evaluation indicators weight by using contribution rate formula and weight determined formula, thus the resulting weight can exclude intervention of human factors and be more objective. Finally, we use TOPSIS method to make comprehensive evaluation rankings for journals.

\subsection{Reduction Algorithm of Evaluation Indicators Based on Equivalence Relation Thought in Rough Set}

Rough set theory extends classical set theory, the knowledge, which is used for classification, is embedded in set as a part. There are some key definitions as follows:

Definition 1: any information systems can be briefly represented as a two-tuples $S=<U, A>$, where $U$ represents a non-empty set attributes; $A$ represents attribute set.

Definition 2: supposing $\operatorname{ind}(B)(B \subseteq A)$ is an equivalence relation on $U$, It produce the equivalence classes: $[x]_{\text {ind }(B)}=\left\{y\left|[x]_{i n d(B)}\right| x \in U\right\}$, the equivalence classes set is denoted by $U \mid \operatorname{ind}(B)=\left\{[x]_{\operatorname{ind}(B)} \mid x \in U\right\}$, can be called a knowledge system on $U$.

Definition 3: supposing $a \in A$, If the property $a$ does not affect the classification of domain of discourse $U$, that is, $U \mid \operatorname{ind}(A)=\{\operatorname{ind}(A-\{a\})\}$, can be called $a$ is redundant in the knowledge system, if not, $a$ is necessary.

Definition 4: supposing $p, s$ are two equivalent relations on $U, U \mid S=\left\{X_{1}, X_{2}, \ldots, X_{n}\right\}$, positive field of $s$ is denoted by $\operatorname{POS}_{p}(s)$, that is, $\operatorname{POS}_{p}(s)=\bigcup_{i=1} p-\left(X_{i}\right)$. If there is $r \in p$, and $\operatorname{POS}_{p}(s)=P O S_{p-(r)}(S)$, be called $r$ can be omitted in $p^{\prime} S$. $p$ - $\{r\}$ is relatively simplified in $p^{\prime} S$.

For the academic level of sci-tech journals evaluation indicators system, where there are different evaluation levels according to different evaluation indicators sets about evaluation object. Base on equivalence relation thought in Rough set theory, and focus on the need to find the necessary evaluation indicators from the evaluation indicators set, making the order of evaluation according to the necessary evaluation indicators can fully consistent with the results according to the all evaluation indicators, enabling effective reduction of evaluation indicators without losing any information.

Base on Rough set theory and reduction principles of the academic level of sci-tech journals evaluation system, we can obtain reduction algorithm of evaluation indicators, described as follows:

Step 1: for the evaluation indicators system $B=\left\{a_{i}\right\}(i=1, \ldots, m)$, obtaining $\operatorname{IND}(B)$.

Step 2: for $i=1, \ldots, m$, obtaining $\operatorname{IND}\left(B-\left\{a_{i}\right\}\right)$.

Step 3: if $\operatorname{IND}\left(B-\left\{a_{i}\right\}\right)=\operatorname{IND}(B)$, that is, $a_{i}$ is miscellaneous indicator can be removed, if not, $a_{i}$ is necessary indicator cannot be removed.

\subsection{The Determination of Indicators Weight Formula Based on Rough Set}

Any one object has a number of indicators attributes, the degree of importance determines the value of the indicators weight in the evaluation. For the method to determine the values of indicators attributes, divided into three categories: One is the subjective weighting method, it takes experience to determine the weight, such as subjective weighting method, the Delphi method, AHP method, Adjacent index comparison method; another one is Objective weighting method, such as, the mean variance method, principal component analysis, the method of maximizing deviations, Entropy value method, and a combination of subjective and objective weighting method (Li Yuan-yuan et al., 2009). Base on Rough set theory, starting from the internal relation of the known data to determine the degree of importance about attributes of the object. According to presentation about indiscernible relation and the importance of indicators 'attributes in Rough set theory, we can obtain the contribution rate formula about indicators to the evaluation results as follows: 


$$
w_{r}=\frac{\left|\operatorname{POS}_{P}(Q)\right|-\left|\operatorname{POS}_{P-\{r\}}(Q)\right|}{U}
$$

Where $U$ is evaluation objects set; $P, Q$ is subset of indicators; $w_{r}$ is contribution rate, which is indicator $r$ to evaluation results

Thus, we can obtain the formula of indicator weight as follows:

$$
\lambda_{r}=\frac{w_{r}}{\sum_{i \in P} w_{i}}
$$

Where $\lambda_{r}$ is indicator weight. (Huang Jian et al., 2009)

\subsection{TOPSIS Method}

TOPSIS method is an excellent non-linear evaluation method, the basic idea is: comprehensive evaluation problem is transformed into gaining the differences between each evaluated object-"distance". That is, be in the normalized data matrix to find the optimal goal and the worst goal and determine the positive ideal solution and the negative ideal solution, then to calculate the distance from each evaluation object to the ideal solution and also the negative ideal solution, and comparing the distances to get comprehensive evaluation rankings.

Concrete steps of TOPSIS method are as follows:

Step 1: Polarity process evaluation indicators to obtain polarity consistent matrix, due to the indicators data of this paper are all very large indicators, it may not be processed.

Step 2: Normalize the evaluation indicators through the range transformation method, the normalized matrix $X=\left(x_{i j}\right)_{m \times n}$ is

$$
x_{i j}=\frac{r_{i j}-\min _{i}\left\{r_{i j}\right\}}{\max _{i}\left\{r_{i j}\right\}-\min _{i}\left\{r_{i j}\right\}}, i=1,2, \ldots m ; j=1,2, \ldots, n
$$

Where $r_{i j}$ is the value of the corresponding journal indicators, $x_{i j}$ is the dimensionless value, and also $x_{i j} \in[0,1], i=1,2, \ldots, m ; j=1,2, \ldots, n$.

Step 3: Construct a weighted decision matrix norms $Z$. Based on weight of each indicator, so

$$
z_{i j}=w_{i j} x_{i j}, i=1,2, \ldots m ; j=1,2, \ldots, n
$$

Step 4: Determine positive ideal solution $z^{+}$and negative ideal solution $z^{-}$.

Suppose the $j$-th attribute value of positive ideal solution $z^{+}$is $z_{j}^{+}$, and the $j$-th attribute value of negative ideal solution $z^{-}$is $z_{j}^{-}$.

so, positive ideal solution is denoted by:

$$
z^{+}=\min _{i}\left\{z_{i j}\right\}, i=1,2, \ldots, m ; j=1,2, \ldots, n
$$

negative ideal solution is:

$$
z^{-}=\max _{i}\left\{z_{i j}\right\}, i=1,2, \ldots, m ; j=1,2, \ldots, n
$$

Step 5: Separately calculate the distance between the $i$-th evaluation object and ideal solution. $d_{i}^{+}$is the distance between the $i$-th evaluation object and positive ideal solution $z^{+}$, and $d_{i}^{-}$is the distance between the $i$-th evaluation object and negative ideal solution $z^{-}$:

$$
d_{i}^{+}=\sqrt{\sum_{j=1}^{4}\left(z_{i j}-z_{j}^{+}\right)^{2}}, i=1,2, \ldots, m, \quad d_{i}^{-}=\sqrt{\sum_{j=1}^{4}\left(z_{i j}-z_{j}^{-}\right)^{2}}, i=1,2, \ldots, m
$$

Step 6: Calculate relative comprehensive evaluation value of each object and rank them.

Comprehensive evaluation value for each evaluation object is: 


$$
b_{i}=\frac{d_{i}^{-}}{d_{i}^{+}+d_{i}^{-}}, i=1,2, \ldots, m
$$

Closeness between the evaluation object and the ideal solution can be obtained at this time, closeness value is between $0-1$, if the value is closer to 1 , indicating that the corresponding evaluation object closer to the optimal level; conversely, the value is closer to 0 , the evaluation object represents the worst level, in this way to be ranked.

\section{Instance Analysis}

\subsection{Reduction of Evaluation Indicators Based on Equivalence Relation Thought in Rough Set}

This paper uses data in references (He De-fang et al., 2013), and selects related evaluation data of academic level of 22 sci-tech journals in the same field (that is biological sciences). Let B1, B2, B3, B4, B5, B6, B8, B9, respectively stand for: Extended total cited version number, extended influence factor, extended immediacy index, extended cited rate, extended number of citing sci-tech journals, extended disciplines impact indicators, extended discipline diffusion index, extend cited half-life, extended $\mathrm{H}$ Index. The evaluation indicators set $\mathrm{B}=$ $\{$ B1, B2, B3, B4, B5, B6, B7, B8, B9\}, thereby establishing an academic level of journal evaluation system and evaluate these sci-tech journals into three grade based on data. It shows in table 1. Due to space limitations, we should abbreviate Sci-tech journals names.

Table 1. Evaluation data of sci-tech journals

\begin{tabular}{ccccccccccc}
\hline Journal Name & B1 & B2 & B3 & B4 & B5 & B6 & B7 & B8 & B9 & grade \\
\hline Lab Ani \& Medi & 451 & 0.327 & 0.04 & 0.82 & 212 & 0.1 & 2.38 & 7.06 & 5 & 1 \\
Sichuan Animal & 1162 & 0.471 & 0.067 & 0.88 & 332 & 0.34 & 3.73 & 5.43 & 4 & 2 \\
Heredity & 2577 & 1.03 & 0.147 & 0.92 & 632 & 0.7 & 7.1 & 6.48 & 10 & 3 \\
Applied Entomology & 2507 & 0.723 & 0.111 & 0.9 & 353 & 0.35 & 3.97 & 7.61 & 10 & 3 \\
Chin J of Compar Medi & 1050 & 0.548 & 0.043 & 0.9 & 432 & 0.26 & 4.85 & 5.02 & 5 & 2 \\
Life Science & 792 & 0.668 & 0.084 & 0.95 & 402 & 0.44 & 4.52 & 4.95 & 8 & 2 \\
Chin Biotech & 1766 & 0.662 & 0.149 & 0.96 & 629 & 0.55 & 7.07 & 5.79 & 9 & 3 \\
Chin J Biochem Mol Biol & 892 & 0.452 & 0.181 & 0.88 & 422 & 0.48 & 4.74 & 5.99 & 5 & 2 \\
J of Experi Ani Chin & 619 & 0.572 & 0.053 & 0.9 & 311 & 0.22 & 3.49 & 5.21 & 6 & 1 \\
J of Cell Bio Chin & 709 & 0.536 & 0.074 & 0.93 & 385 & 0.38 & 4.33 & 5.12 & 5 & 1 \\
R of Wild Plants Chin & 1423 & 0.693 & 0.033 & 0.98 & 494 & 0.25 & 5.55 & 8.25 & 8 & 2 \\
Chin J of Mycol & 412 & 0.719 & 0.076 & 0.78 & 162 & 0.09 & 1.82 & 3.81 & 5 & 1 \\
Bulletin of Biol & 1627 & 0.352 & 0.046 & 0.94 & 692 & 0.54 & 7.78 & 8.79 & 8 & 3 \\
J of Biol & 1143 & 0.622 & 0.067 & 0.95 & 554 & 0.53 & 6.22 & 5.65 & 7 & 2 \\
Chemi of Life & 712 & 0.264 & 0.025 & 0.96 & 411 & 0.44 & 4.62 & 7.55 & 5 & 1 \\
life Sci & 719 & 0.434 & 0.06 & 0.97 & 429 & 0.39 & 4.82 & 5.43 & 5 & 1 \\
Life Sci Research & 501 & 0.368 & 0.078 & 0.97 & 319 & 0.3 & 3.58 & 6.81 & 5 & 1 \\
Ecol Sci & 979 & 0.254 & 0.026 & 0.97 & 373 & 0.29 & 4.19 & 7.93 & 7 & 2 \\
Prog in Bioche \& Biophy & 1746 & 1.183 & 0.342 & 0.85 & 660 & 0.52 & 7.42 & 6.12 & 9 & 3 \\
Biotechnology & 1246 & 0.537 & 0.039 & 0.97 & 502 & 0.46 & 5.64 & 6.39 & 6 & 2 \\
Biotechnol Bulletin & 1561 & 0.532 & 0.07 & 0.95 & 522 & 0.48 & 5.87 & 3.88 & 6 & 3 \\
Acta Biophysica Sinica & 490 & 0.565 & 0.083 & 0.94 & 336 & 0.27 & 3.78 & 6.54 & 5 & 1 \\
\hline
\end{tabular}

Due to the different dimensions of evaluation indicators, in order to be able to compare the data, we need to eliminate the dimension of the data, there are some common ways to eliminate dimensions, such as, the range transformation method, standardized method, method of mean value, etc. In order to make the data in [0,1].we adopt the range transformation method(Specific methods as 2.3, step 2 ) to do with data in Table 1, and the results (portion) shown in Table 2.

Table 2. The results (portion) of normalized indicators

\begin{tabular}{cccccccccc}
\hline Journal Name & B1 & B2 & B3 & B4 & B5 & B6 & B7 & B8 & B9 \\
\hline Lab Ani\& Medi & 0.02 & 0.08 & 0.05 & 0.20 & 0.31 & 0.02 & 0.09 & 0.65 & 0.17 \\
Sichuan Animal & 0.35 & 0.23 & 0.13 & 0.50 & 0.48 & 0.41 & 0.32 & 0.33 & 0.00 \\
Heredity & 1.00 & 0.84 & 0.38 & 0.70 & 0.91 & 1.00 & 0.89 & 0.54 & 1.00 \\
Applied Entomology & 0.97 & 0.50 & 0.27 & 0.60 & 0.51 & 0.43 & 0.36 & 0.76 & 1.00 \\
Chin J of Compar Medi & 0.29 & 0.32 & 0.06 & 0.60 & 0.62 & 0.28 & 0.51 & 0.24 & 0.17 \\
\hline
\end{tabular}

In order to use Rough set theory to calculate the data in Table 2, we should first discretize data in table 2, there, we use equidistant partitioning algorithm for data in Table 2 to integer. Considering the number of sci-tech journals, evaluation indicators is small, the data in table 2 of this article will be divided into three discrete intervals. Specific methods: Index is not more than 0.3 , a standardized data set to 1 , index is greater than 0.3 , no 
greater than 0.6 , a standardized data set to 2 , index is greater than 0.6 , a standardized data the data set to 3 . The results (portion) of discretization shown in Table 3.

Table 3. The results (portion) of discretization

\begin{tabular}{cccccccccc}
\hline Journal Name & B1 & B2 & B3 & B4 & B5 & B6 & B7 & B8 & B9 \\
\hline Lab Ani\& Medi & 1 & 1 & 1 & 1 & 2 & 1 & 1 & 3 & 1 \\
Sichuan Animal & 2 & 1 & 1 & 2 & 2 & 2 & 2 & 2 & 1 \\
Heredity & 3 & 3 & 2 & 3 & 3 & 3 & 3 & 2 & 3 \\
Applied Entomology & 3 & 2 & 1 & 2 & 2 & 2 & 2 & 3 & 3 \\
Chin J of Compar Medi & 1 & 2 & 1 & 2 & 3 & 1 & 2 & 1 & 1 \\
\hline
\end{tabular}

Finally, according to reduction algorithm of evaluation indicators based on equivalence relation thought in Rough set, write a MATLAB program to solve it, after reduction to get six core evaluation indicators: Extended influence factor, extended immediacy index, extended cited rate, extended number of citing journals, extend cited half-life, extended H Index. That is, the order of evaluation according to the core evaluation indicators can fully consistent with the results according to the all evaluation indicators.

\subsection{The Determination of Core Indicator Weigh Based on the Degree of Importance of Attribute}

After reducing, we extract the core academic evaluation indicators of sci-tech journals. Let A1, A2, A3, A4, A5, A6, respectively stand for: Extended influence factor, extended immediacy index, extended cited rate, extended number of citing journals, extend cited half-life, extended $H$ Index. The core evaluation indicators set $A=\{A 1$, A2, A3, A4, A5, A6 \}, thereby establishing an academic level of journal evaluation system. Given the Rough set theory can only be applied to the regular discrete data, we should first discretize data in the system (specific methods as 3.1), and the results shown in Table 4 (sci-tech journals number in table 4 and order of sci-tech journals in table1 are the same) below.

Table 4. The results of discretization

\begin{tabular}{cccccccccccccc}
\hline J number & A1 & A2 & A3 & A4 & A5 & A6 & J number & A1 & A2 & A3 & A4 & A5 & A6 \\
\hline 1 & 1 & 1 & 1 & 2 & 3 & 1 & 12 & 2 & 1 & 1 & 1 & 1 & 1 \\
2 & 1 & 1 & 2 & 2 & 2 & 1 & 13 & 1 & 1 & 3 & 3 & 3 & 3 \\
3 & 3 & 2 & 3 & 3 & 2 & 3 & 14 & 2 & 1 & 3 & 3 & 2 & 2 \\
4 & 2 & 1 & 2 & 2 & 3 & 3 & 15 & 1 & 1 & 3 & 2 & 3 & 1 \\
5 & 2 & 1 & 2 & 3 & 1 & 1 & 16 & 1 & 1 & 3 & 3 & 2 & 1 \\
6 & 2 & 1 & 3 & 2 & 1 & 3 & 17 & 1 & 1 & 3 & 2 & 2 & 1 \\
7 & 2 & 2 & 3 & 3 & 2 & 3 & 18 & 1 & 1 & 3 & 2 & 3 & 2 \\
8 & 1 & 2 & 2 & 3 & 2 & 1 & 19 & 3 & 3 & 2 & 3 & 2 & 3 \\
9 & 2 & 1 & 2 & 2 & 1 & 2 & 20 & 1 & 1 & 3 & 3 & 2 & 2 \\
10 & 1 & 1 & 3 & 2 & 1 & 1 & 21 & 1 & 1 & 3 & 3 & 1 & 2 \\
11 & 2 & 1 & 3 & 3 & 3 & 3 & 22 & 2 & 1 & 3 & 2 & 2 & 1 \\
\hline
\end{tabular}

We use data in table 4 and the determination of indicator weight formula based on Rough set can determine weight of core evaluation indicators as follows: (numbers in the following formulas stand for sci-tech journals numbers in table 4)

$$
\begin{aligned}
& U|\operatorname{ind}(A-\{A 1\})|=\{1,2,(3,7), 4,5,6,8,9,10,11,12,(13,20), 14,15,16,(17,22), 18,19,21\} \\
& U|\operatorname{ind}(A-\{A 2\})|=\{1,2,3,4,5,6,7,8,9,10,11,12,13,14,15,16,17,18,19,20,21,22\} \\
& U|\operatorname{ind}(A-\{A 3\})|=\{(1,15),(2,17), 3,4,5,6,7,8,9,10,11,12,13,14,16,18,19,20,21,22\} \\
& U|\operatorname{ind}(A-\{A 4\})|=\{1,2,3,4,5,6,7,8,9,10,11,12,13,14,15,(16,17), 18,19,20,21,22\} \\
& U|\operatorname{ind}(A-\{A 5\})|=\{1,2,3,4,5,6,7,8,9,10,11,12,13,14,(15,17), 16,18,19,(20,21), 22\} \\
& U|\operatorname{ind}(A-\{A 6\})|=\{1,2,3,4,5,6,7,8,9,10,11,12,13,14,(15,18),(16,20), 17,19,21,22\}
\end{aligned}
$$

Then by equation 2 and 3 each to calculate contribution rate and weight of core evaluation indicators:

$$
\begin{aligned}
& w_{1}=\frac{4}{23}, w_{2}=\frac{1}{23}, w_{3}=w_{6}=w_{5}=\frac{3}{23}, w_{4}=\frac{2}{23} \\
& \lambda_{1}=\frac{1}{4}, \lambda_{2}=\frac{1}{16}, \lambda_{3}=\lambda_{5}=\lambda_{6}=\frac{3}{16}, \lambda_{4}=\frac{1}{8}
\end{aligned}
$$

Thus, we can determine the weight of each core evaluation indicators of sci-tech journals. That is, extended 
influence factor, extended immediacy index, extended cited rate, extended number of citing journals, extend cited half-life, extended H Index are respectively $\frac{1}{4} 、 \frac{1}{16} 、 \frac{3}{16} 、 \frac{1}{8} 、 \frac{3}{16} 、 \frac{3}{16}$.

As can be seen from the results, extended influence factor has the heaviest weight in core evaluation indicators. In actual academic level evaluation, influence factor related evaluation indicators show great impact on academic evaluation. Therefore, Rough set theory can fully mining the intrinsic link of data obtained, does not require previous experience, thus eliminating the subjectivity of human intervention, making the evaluation more objective, but also weight-determined does not lose accuracy.

\subsection{Academic Ranking of Sci-tech Journal Based on TOPSIS}

This section, we use an excellent non-linear evaluation method-TOPSIS to rank for sci-tech journals. Firstly, we use the basic steps of TOPSIS method to do with data in table1, and then combined with the core indicators weights obtained in the previous section, to calculate comprehensive evaluation value (TOPSIS value) of journals using MATLAB programming. When the comprehensive evaluation value closer to 1 , that is described the state of journal closer to the optimal state, on the contrary, when the comprehensive evaluation value closer to 0 , indicating the state of this journal closer to the worst state. Finally, according to the comprehensive evaluation value for 22 academic sci-tech journals to rank, get their comprehensive ranking in table 5 .

Table 5. Comprehensive ranking

\begin{tabular}{cccccc}
\hline Journal Name & TOPSIS & ranking & Journal Name & TOPSIS & ranking \\
\hline Heredity & 0.596 & 1 & Life Sci Research & 0.367 & 12 \\
Prog in Bioche \& Biophy & 0.584 & 2 & life Sci & 0.357 & 13 \\
R of Wild Plants Chin & 0.537 & 3 & Biotechnol Bulletin & 0.336 & 14 \\
Bulletin of Biol & 0.524 & 4 & Acta Biophysica Sinica & 0.311 & 15 \\
Chin Biotech & 0.495 & 5 & Chin J Biochem Mol Biol & 0.241 & 16 \\
Applied Entomology & 0.490 & 6 & Chin J of Compar Medi & 0.235 & 17 \\
Ecol Sci & 0.429 & 7 & J of Cell Bio Chin & 0.233 & 18 \\
Biotechnology & 0.401 & 8 & J of Experi Ani Chin & 0.222 & 19 \\
J of Biol & 0.385 & 9 & Lab Ani\& Medi & 0.192 & 20 \\
Chemi of Life & 0.376 & 10 & Sichuan Animal & 0.183 & 21 \\
Life Science & 0.373 & 11 & Chin J of Mycol & 0.132 & 22 \\
\hline
\end{tabular}

Table 5 shows the comprehensive ranking of 22 sci-tech journals, including the Heredity, Prog in Bioche\& Biophy, R of Wild Plants Chin, Bulletin of Biol, Chin Biotech, Applied Entomology rank the top six in these 22. In all of them, according to reference(Anonymity,2009)and some other information from Internet, we know that Heredity, Prog in Bioche \& Biophy, R of Wild Plants Chin, Bulletin of Biol, Chin Biotech, Applied Entomology of these sci-tech journals in the biological sciences field also enjoy a relatively high reputation, ranking the relatively top. It can be determined that the academic level of sci-tech journals evaluation model based on Rough sets and TOPSIS achieved good results, the result is consistent with the actual situation, but also proved the feasibility and effectiveness of the model.

\section{Conclusion}

Based on Rough set theory and TOPSIS method for sci-tech journals evaluation can have a fully data mining, providing a model for a more objective academic evaluation of sci-tech journals.

Firstly, according to evaluation indicators of 22 sci-tech journals, to establish evaluation indicators system, and then establish reduction algorithm of evaluation indicators based on equivalence relation thought in Rough set, and we should use the range transformation method and equidistant partitioning algorithm to do with data, what' more, programming to obtain core evaluation indicators. In this way, the advantage is that the removal of evaluation indicators and the evaluation work can be more efficient.

Afterwards, according to core evaluation indicators previously obtained, to establish core evaluation indicators system, and then using the determination of indicator weight formula to calculate the weight of each core evaluation indicators. Such processing cans fully mining the internal relation of data and excludes the intervention of human factors, more objectivity.

Finally, we adopt non-linear evaluation method (TOPSIS) to make a comprehensive evaluation of sci-tech journals to rank, using evaluation indicators data obtained in this paper as well as the weight determined, and then programming to obtain the closeness between each evaluation object and ideal solution, and also comprehensive evaluation ranking. 
This paper, based on Rough set theory and TOPSIS method for 22 sci-tech journals to conduct comprehensive evaluation rankings, fully mining the intrinsic link of data obtained, reflecting the objectivity of the model. However, the data in this paper is relatively simple, no incomplete data, but in the actual evaluation, Rough set theory can be used to improved limited tolerance relation to handle null values indicators, to make the model more accurate.

Rough set theory and TOPSIS method is widely used in the field of evaluation, but in the future, we can have an in-depth study. The hybrid of the two methods not only can be used in academic evaluation, also can be applied to other fields, such as, atmospheric environmental quality assessment, short-term wind speed forecasting, etc.

\section{Acknowledgements}

The authors acknowledge the financial support of this research by the Key Laboratory of Product Packaging and Logistics of Guangdong Higher Education Institutes, the Fundamental Research Funds for the Central Universities, the project of the Natural Science Foundation of Guangdong Province (No. S2012010008773), the projects of Zhuhai Science, Technology, Industry, Trade and Information Technology Bureau (No. 2011B050102013 \& 2012D0501990033), the project of the college students' innovation and entrepreneurship training plan in Jinan university (national level) (No. 201410559004).

\section{Reference}

Anonymity. (2009). List of biological science in Chinese core journals. Heredity, 4, 406-406.

Cao, Z. G., \& Chai, C. H. (2007). Application of Fuzzy Multilevel Comprehensive Evaluation Method in Assessment of Scientific Research Subjects. Aeronautical Computing Technique, 1, 51-52.

Chen, H. Z. (2003). Principal component analysis applied in the evaluation of academic journals. Journal of Inner Mongolia Normal University(Natural Science Edition), 12, 38-39.

He, D. F., etc. (2013). 2013 China's science and technology journal citation reports (KuoKan version). China, BeiJing: Science and technology literature press.

Huang, J., Zhang, Y. B. (2009). Rough set theory in the application of scientific research ability of comprehensive evaluation. Science and Technology Management Research, 2, 83-84.

Li, Y. Y. (2009). Research on the Construction of Indicator System and Comprehensive Evaluation Method Based on Rough set (Doctoral dissertation). Wuhan University of Technology, Wuhan, China.

Lin, C. Y., \& Mo, L. (2004). A tribute Mathematical Model for Comprehensive Index System' Quality Evaluation for Natural Science Academic Journals. Mathematics in practical and theory, 5, 1-7.

Tao, S. J. (2013). Combination Empowerment-TOPSIS Method Based on the Application Research of Real Estate Investment Decision-making Model (Master's thesis), Chongqing: Chongqing University, Chong Qing, China.

Wang, G. Y., Yao, Y. Y., \& Yu, H. (2009). A Survey on Rough set Theory and Applications. Chinese Journal of Computer, 7,103-108.

Wang, L. Z., \& Yu, L. P. (2012). Study on Key Indicators Definition and Its Impact in Evaluation of Academic Journals. New Technology of Library and Information Service, 7(8), 1229-1246.

Wang, L. L., Lu, X. N., \& Sun, Z. Gao. (2014). Application of improved topsis in atmospheric environmental quality assessment. Environmental Engineering, 09, 142-146.

Wang, Y. Y., Zhang, Z., \& Wang, Z. X. (2011). An Improved Weighted TOPSIS Method and Its Application Based on Grey Relational Coefficient. East China Economic Management, 10, 139-144.

Xiao, J. H., \& Wu, J. P. (2001). The Evaluation Method for Science Research Project based on Rough set. Science Research Management, 9, 8-12.

Yin, D. Y., Sheng, Y. F., Jiang, M. J., Li, Y. S., \& Xie, Q. T. (2014). Short-term wind speed forecasting using Elman neural network based on rough set theory and principal components analysis. Power System Protection and Control, 11, 46-51.

\section{Copyrights}

Copyright for this article is retained by the author(s), with first publication rights granted to the journal.

This is an open-access article distributed under the terms and conditions of the Creative Commons Attribution license (http://creativecommons.org/licenses/by/3.0/). 